\title{
ADVANCING SIMULATION EXPERIMENTATION CAPABILITIES WITH RUNTIME INTERVENTIONS
}

\author{
Joon-Seok Kim \\ Hamdi Kavak
Dept. of Geography \& Geoinformation Science
George Mason University
4400 University Drive
Fairfax, VA, USA
\{jkim258, hkavak\}@gmu.edu

Umar Manzoor

Dept. of Computer Science

Tulane University

6823 St. Charles Ave.

New Orleans, LA, USA

umanzoor@tulane.edu

\author{
Andreas Züfle \\ Dept. of Geography \& Geoinformation Science \\ George Mason University \\ 4400 University Drive \\ Fairfax, VA, USA \\ azufle@gmu.edu
}

\begin{abstract}
Experimentation is a critical capability of simulations that allows one to test different scenarios safely and cost-effectively. In particular, agent-based simulations have been used in experimenting with different policy options to aid decision makers. Highly utilized experimentation methods such as parameter sweeping aim to explore the relationship between the initial parameter values (i.e., input) and simulation results (i.e., outputs). Experimentation, which involves changes of simulation states on-the-fly, is often conducted adhoc and entails manual code adjustments which are time consuming and error-prone. In this paper, we present a framework that facilitates intervening in a running simulation to change simulation states in a semi-automated manner so that a simulation user can explore alternative worlds. In our framework, such an intervention is implemented using an injection mechanism. The framework allows the user to weigh different policy options rapidly with minimal effort. We illustrate its use in an urban agent-based model.
\end{abstract}

Keywords: injection, intervention, checkpoint, experimentation, what-if analysis

\section{INTRODUCTION}

One of the grand challenges of social scientists is the lack of objective knowledge of the actual causes of observed behaviors in the real world. Conducting the necessary experimental work for understanding causality in social behaviors and systems is often impractical or unethical, while observational modeling, data mining and machine learning approaches struggle to infer causality from correlation patterns. Planners and decision-makers often rely upon agent-based simulation to help them understand and forecast a variety of scenarios that involve complex human social systems and behaviors. In particular, decision-makers often 
seek to identify, characterize, and model causal processes at different scales and for different social systems to help explain or predict certain patterns of behavior for a wide range of applications, including disease spread (Carley et al. 2006, Perez and Dragicevic 2009, Crooks and Hailegiorgis 2014), crime and riots (Malleson, Heppenstall, and See 2010, Pires and Crooks 2017), and economic systems (Farmer and Foley 2009).

An agent-based model (ABM) is a computerized simulation of individual entities called agents, which interact through prescribed rules in a well-defined environment. The goal in designing an ABM is to abstract complex real-world human behavior into a finite set of realistic rules leading to a system of socially plausible behavior. This goal can be achieved through careful validation and verification of the model, using sensitivity analysis (Kleijnen 2005) and model calibration (Lamperti, Roventini, and Sani 2018).

Once an ABM has been implemented, validated, verified and calibrated for a particular application, it can be used to experimentally evaluate new policies, unexpected events, and disruptive changes to the simulation environment. For instance, in an economic system ABM new economic policies can be employed. Such "what-if" scenario explorations allow us to observe outcomes of policy changes in settings that are too complex to describe by analytic models. What-if analysis is a crucial process used to diagnose a model, analyze its features, and pinpoint a circumstance for better decision making. In general terms, such scenario explorations are realized by (1) changing the initial conditions of the simulation and (2) making changes during runtime. While both allow realizing what-if analyses, their implementation and exploration capabilities are vastly different.

Changing the initial conditions of a simulation, also known as initialization, is a more common approach to scenario explorations. Grow (2017) proposed the use of a regression metamodel to facilitate the understanding of the complex behaviors in agent-based simulations. This type of metamodel is quite effective for computational demography as it is highly accessible and easy to communicate. Thiele, Kurth, and Grimm (2014) proposed the RNetLogo package by linking NetLogo (widely used toolkit for agent-based modeling) and R (statistical analysis tool) to use the established methods to develop tools to facilitate parameter estimation and sensitivity analysis in agent-based models. Concurrently, Ligmann-Zielinska et al. (2014) proposed a simulation framework based on quantitative uncertainty and sensitivity analyses to develop parsimonious socio-ecological agent-based models to understand model behavior and explore the outcome space.

Further, many popular agent-based modeling platforms, such as NetLogo and AnyLogic, provide standardized ways for users to specify parameter value combinations and replications. This allows users to run multiple scenario alternatives with minimal effort. On the other hand, such runs can only support narrow explorations due to limiting the changes at the initialization level.

Researchers developed alternative mechanisms to increase experimentation capabilities of simulations. One popular technique for runtime explorations is called "cloning" (Li, Cai, and Turner 2017). Simply put, cloning makes a copy of a simulation while it's running and allows the cloned instance to run semiindependently (Hybinette and Fujimoto 1997). Majority of contributions in this line of research come from parallel simulation community that aims to create massive-scale models and explore runtime changes (Hybinette and Fujimoto 1997, Hybinette and Fujimoto 2001, Yoginath and Perumalla 2018). While these studies provide efficient ways to share computational resources with cloned instances, they heavily rely on running simulations on high-performance computing infrastructures (Yoginath and Perumalla 2018). Many ABM platforms and open source tools do not support high-performance computing infrastructures making them inaccessible to a wider community of simulation modelers. That is where our study comes into play.

In this work, we propose a framework to prescribe changes into a running $\mathrm{ABM}$ to support semi-automated "what-if" analyses. Our framework provides an interface for existing ABMs, which allows a user to define checkpoints in a running simulation. At these checkpoints, changes to model parameters can be prescribed, and the checkpoint can be loaded at a later time to compare outcomes of different strategies and policies 
in alternative simulation time-lines originating from the same checkpoint differing merely in the prescribed changes. Our framework allows to define conditions for simulation checkpoints (such as the occurrence of a disaster or the observed outbreak of a disease), and allows to define multiple policies to intervene the running simulation (such as defining different solutions to prevent the spread of a disease), which may be defined by a human expert based on information available at the time of the simulation checkpoint. Once defined, the prescribed changes are automatically injected into the simulation. In addition to our injection mechanism, we describe our injection builder for user-friendly analysis of the current simulation state and for allowing the user to register prescriptions to the model and their conditions. We further demonstrate the effectiveness of our framework using a practical use-case based on an ABM that simulates patterns of life in an urban environment to study the spread of influenza-like disease. We evaluate various intervention scenarios based on vaccinations, closures of public places, and employment of a stay-home-order that we inject into our simulation.

\section{FRAMEWORK FOR SIMULATION EXPERIMENTATION}

Experimentation is a significant process to collect scientific evidence to support a hypothesis. It requires, however, tremendous time and effort to not only 1) conduct experiments themselves but also 2) prepare them. This section introduces a framework for simulation experimentation, focusing on how to expedite the process of experimentation. Fig. 1 shows our proposed process of experimentation. It is an iterative process of simulation of a model, intervention, and examination. Note that the model in Fig. 1 is a tested and certified computational model that went through the validation and verification process; thus no change of model is needed. In this paper, intervention denotes an intended action to intervene between simulation and an analyst to achieve the altered outcome in hopes of resolving scientific questions. To decide whether an analyst will proceed more experimentation is required after examination of collected results. In what follows, the main loop is explained in detail.

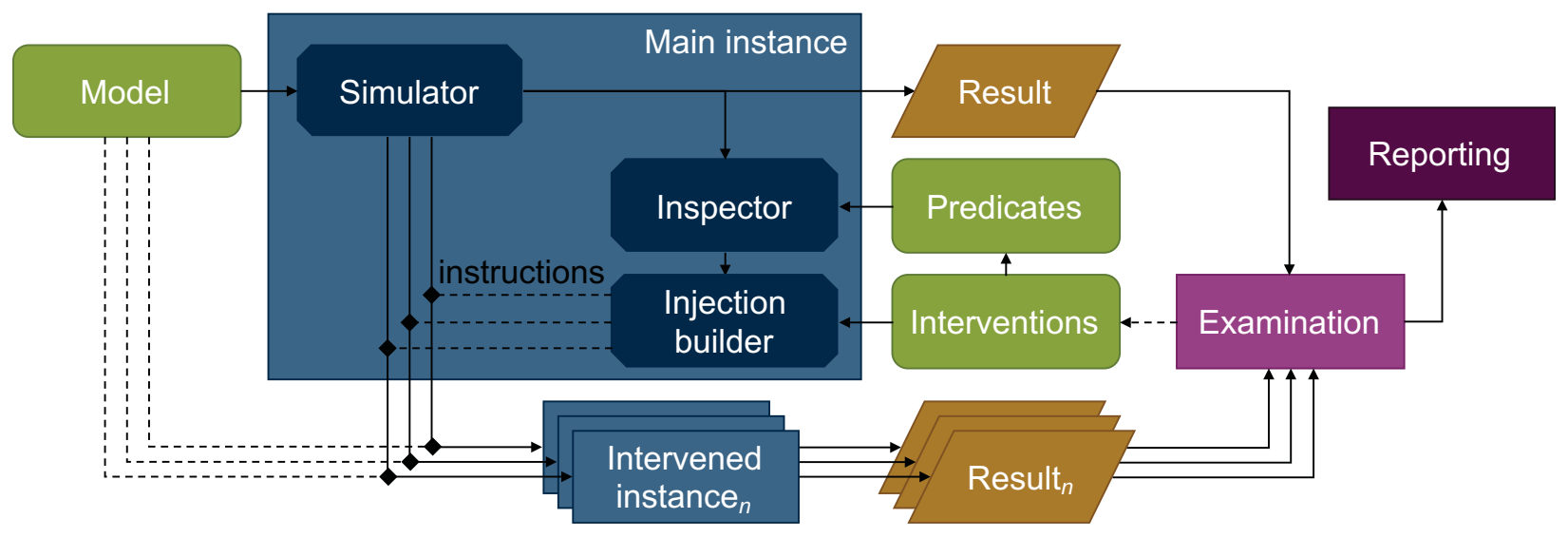

Figure 1: Process of experimentation.

\subsection{Instruction Injection}

Dependency injection is a software design pattern that allows modules to be loosely coupled (Prasanna 2009). It enables a client to configure behaviors without explicit code changes, providing the flexibility of configuration. Similarly, we adopt an injection mechanism as the implementation of interventions. We interpret the content of injection as an instruction, a procedure that may affect simulation and produce different results of the simulation. Instruction injection is an act to inject instructions into the simulation. 
It allows an analyst to do experimentation without altering models and simulators at the code level. This mechanism facilitates what-if analysis with less effort so that one can focus on their experimentation and analysis. From an analyst's perspective, to temporarily close public places to see how the decision impacts simulation is an example of interventions (see Section 3).

\subsection{Injection Builder}

From an implementation perspective, an instruction manipulates values and invokes a series of functions dynamically. To inject instructions into a running simulation, it is necessary to define an interface or injection point. Namely, instruction injection requires a pair of an instruction and a point when the instruction is triggered. This point can be a specific simulation time or a transition between states depending on paradigms in simulation modeling. Fig. 2 illustrates an injection point between $s_{t}$ and $s_{t+1}$ and the differentiation of simulation occurs from $s_{t+1}$ with intervention $\delta$.

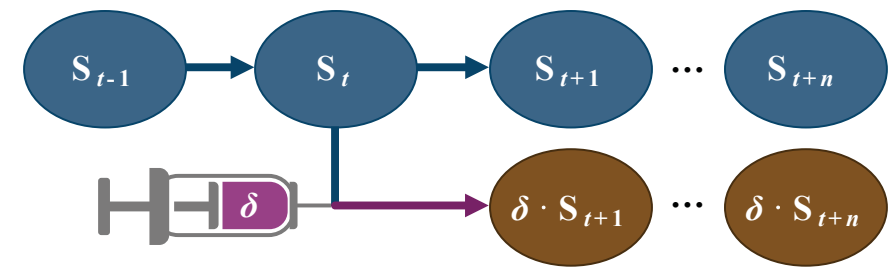

Figure 2: Influence of intervention.

It is paramount to minimize the effort of an analyst. For this purpose, we have designed an injection builder which is a tool that facilitates the creation of instruction injection in a semi-automated or automated way. In the initial phase of simulation, an analyst might not recognize phenomena interesting to them unless they see tangible results. Once they identify their targets to do experimentation, a task to define predicates of interest is given. A predicate is a description of the condition to produce injection points. For instance, to check if the number of infected agents in a disease spread ABM is greater than five percent of the population can be a predicate. Our inspector module enables users to interact with simulation and carry out this task. We note that for the task no change in code level is involved. If an analyst is aware of targets and injection points beforehand, the inspection process is not required. We formally define an intervention as follows.

Definition 1 (Intervention). Intervention $\delta$ is a pair of $(t, \lambda)$, where $t$ is an injection time and $\lambda$ is a set of instructions.

\subsection{Implementation}

This subsection addresses the implementation of our framework including the inspector, injection builder, and intervention agent (see Fig. 3). We implemented the framework based on MASON (Luke, Cioffi-Revilla, Panait, Sullivan, and Balan 2005), a multiagent simulation library core in Java. Although MASON provides inspectors that inspect properties, they are not designed to evaluate predicates during runtime. Thus, we developed modules to dynamically evaluate predicates given by users. There is a trade-off between flexibility and performance of dynamic evaluation. That is, dynamic evaluation allows to check undefined predicates, but it entails subsidiary operations such as parsing predicates and searching methods. Therefore, handling processed data such as statistical data at the code level is preferable to specifying detailed predicates. The augmented inspectors are helpful when an analyst wants to pause simulation at the time when user-defined conditions are met. In this setting, inspectors are used to pinpointing an injection time. If an analyst is aware of injection timing, they can create intervention using the injection builder without explicit inspection. It 
also allows creating a checkpoint of simulation on the fly. A checkpoint is used to restore the simulation to the checkpoint for analysis of alternative timelines. Moreover, it expedites the experimentation process because it is not required to simulate from the beginning.

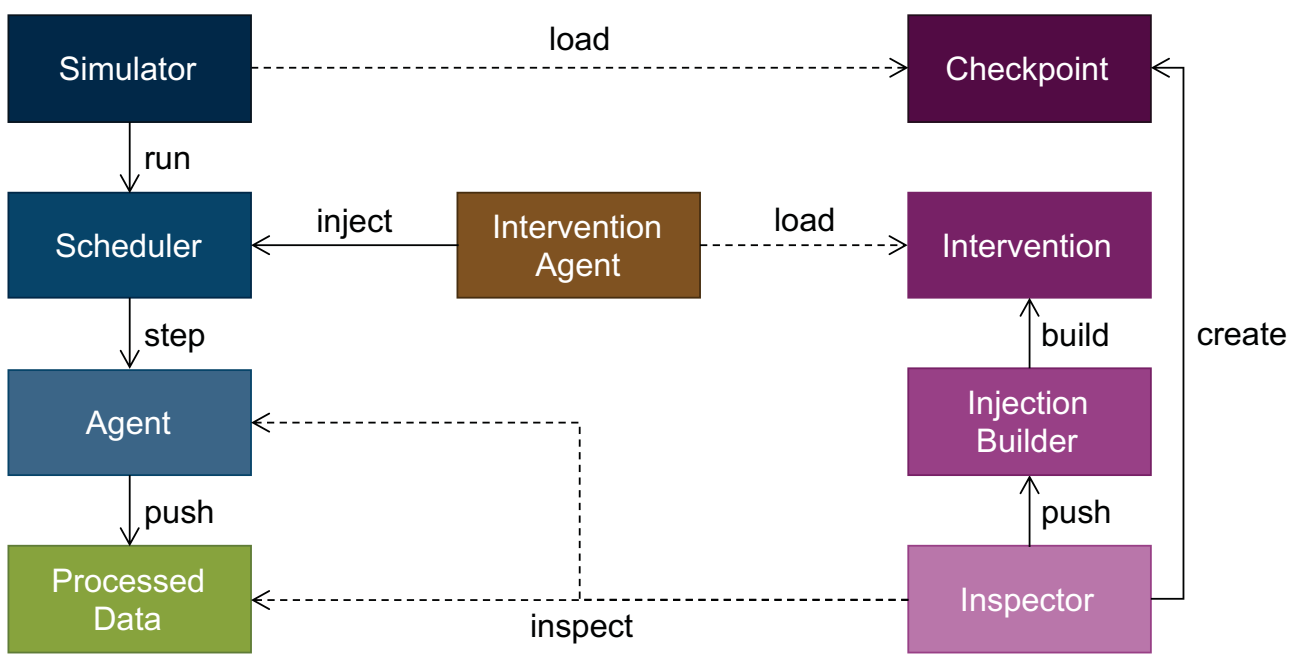

Figure 3: Conceptual architecture of framework.

The injection builder is a library that facilitates performing instructions, namely invoking public methods defined by users. It enables to generate various combinatorial test cases. As a result of building, the injection builder produces an intervention file that can be loaded from a simulator. The file contains intervention information such as time, access methods, and parameters corresponding to each method. To implement the injection mechanism, we introduced an intervention agent which is an agent to maintain schedules of interventions without disturbing user-defined agents. Restoring simulation from a checkpoint, the intervention agent loads intervention files and schedules instructions.

\section{USE CASE}

To illustrate the usability of our framework, we created an agent-based urban model that simulates the patterns of life with the goal to investigate the spread of influenza-like diseases. In this model, agents are situated on a synthetic geographic area created based on procedural city generation techniques (Kim, Kavak, and Crooks 2018). When it comes to behaviors, agents follow simple routines such as eating, working, commuting, and visiting recreational facilities. In the real-world as well as in our simulated world, such facilities serve as the hub for spreading contact/proximity-based diseases. Thus, disease spread is a useful example to illustrate the important capabilities of our framework.

Disease models have been heavily investigated using compartmental mathematical models. In these models, a population is divided into compartments based on disease stages, each compartment is then represented as a state, and changes in these states are captured using simple rate-based differential equations. However, such an approach assumes that all members of a compartment are homogeneous in their characteristics and their spatial locations are not captured. This makes it challenging to represent populations realistically and to develop reliable intervention scenarios. Agent-based disease models have the potential to capture the heterogeneity of populations thanks to its bottom-up modeling approach. Such models have been used for many critical purposes including public health policy development (Apolloni et al. 2009, Kumar et al. 2013), investigation of biological virus evolution (Roche, Drake, and Rohani 2011), and forecasting (Nsoesie et al. 2013), etc. Here our purpose is not to create a novel disease model, but to show the applicability of our framework in a critical domain. 


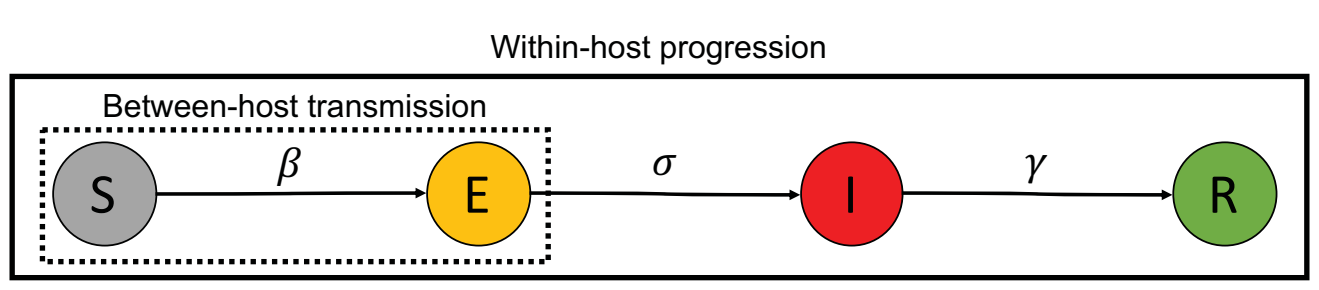

Figure 4: A depiction of a disease transmission process with transition rate parameters.

\subsection{Disease Model}

We based our disease model on a well-known Susceptible-Exposed-Infectious-Recovered (SEIR) approach where each of these letters represents different stages of an epidemic disease (Riley 2007). Here, $S$ indicates that the person is Susceptible to infection but not infected yet, $E$ represents a latent state for disease where the person is Exposed to the disease but not infectious yet, $I$ represents the disease state where the person is Infectious and can also infect other people, and finally $R$ represents Recovered, the disease stage when the person is immune to the disease either through vaccine or recovery. Following Barrett et al. 2008, we constructed two stages of disease model: within-host progression and between-host transmission. A simple depiction of this model is illustrated in Fig. 4.

The within-host progression part of the model deals with the life-cycle of the disease within an individual. In other words, disease progression is represented as a finite state machine with specific transition probabilities. All individuals are assumed to be starting with $\operatorname{Susceptible(} S$, i.e., prone to getting infected). Unless an individual gets the virus, $S$ disease stage stays the same. Once the virus is in the body, the disease stage immediately becomes Exposed $(E)$ and stays like that for a certain time which is captured using the transition rate $\sigma$. Then, the disease progresses to become Infectious $(I)$. Similarly, this stage continues for a certain time and the person becomes Recovered $(R)$ which is captured using the transition rate $\gamma$. We assume that people who are Recovered will be immune to the disease regardless of their future contacts with infectious individuals.

The between-host transmission part of the disease model deals with the infection of a susceptible individual through meeting with at least one Infectious (I) individual. Such meetings have the probability of transmitting the virus from infectious individuals to susceptible individuals through contact or being at close proximity. Following Barrett et al. 2008, we use the between-host transmission probability formulated in Equation (1) for any individual $i$ who are susceptible.

$$
\beta=1-\exp \left(\tau \sum_{k \in K} N_{k} \ln \left(1-k s_{i} \rho\right)\right)
$$

Here, $\tau$ is the duration of contact with infectious individuals, $N_{k}$ is the number of infectivities with $k$ from the set of all infectivities $K, s_{i}$ is the susceptibility of the individual, and $\rho$ is the probability that one susceptible individual gets infected by one infectious individual during one minute of contact. We compute $\rho$, a diseasespecific property, using the formula $R_{0}=\rho / \gamma$, where $R_{0}$ is the reproduction number for the disease. We assume that all non-infected individuals have the same susceptibility $s=1$ and all infectious individuals have the same infectivity $k=1$. Thus the probability $\beta$ simply becomes $\beta=1-\exp (\tau N \ln (1-\rho))$, where $N$ is the number of infectious individuals. To use these parameters in a realistic way, we follow Balcan et al. 2009 who estimated reproduction number $R_{0}$ as well as transition rates $\sigma$ and $\gamma$. All these parameters and their values are summarized in Table 1. 
Table 1: Disease parameter values used in the model.

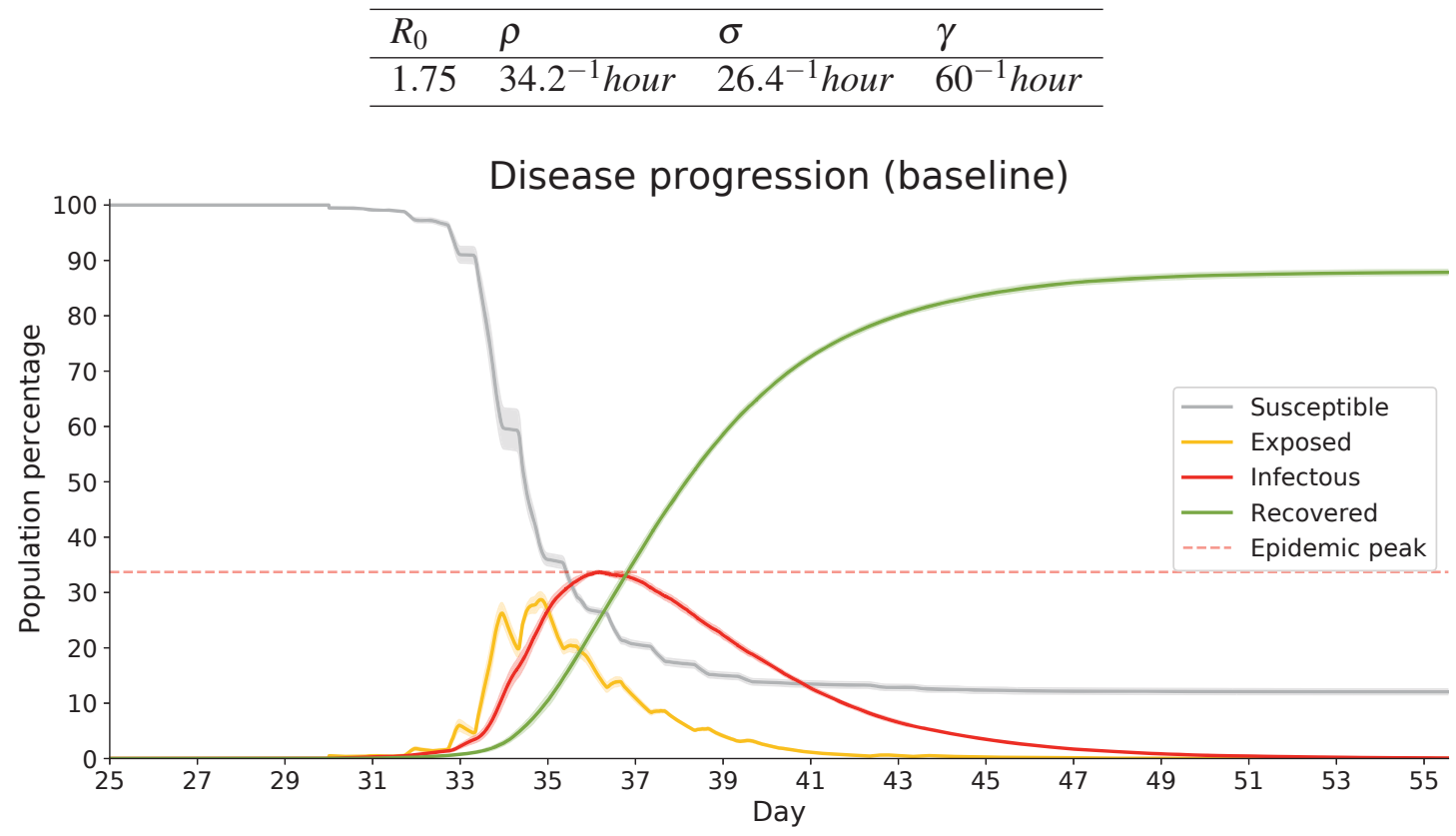

Figure 5: Baseline disease spread progression over ten replications. Each color represents a different percentage of disease stages within the population. The solid lines indicate the average values while shaded areas show $95 \%$ confidence band.

\subsection{Simulation Setup, Intervention Scenarios, and Results}

We populated a small-scale synthetic city with 6,000 inhabitants and added various places including workplaces, homes, restaurants, and other venues (e.g., recreational places). Agents follow their daily activities such as working when it is a work day, eating when feeling hungry, and visiting venues. Each simulation step represents 5 minutes in the real world time. We start the simulation at day 0 and run it for over 60 days while the first 25 days are considered warm-up period for agents to establish their networks. At the beginning of day 30 , we introduce $0.5 \%$ of the population a hypothetical influenza-like virus. Fig. 5 shows our results for the baseline runs with the introduction of the virus. The most critical result is that, around day 36, the population reaches its highest percentage of Infectious people $(\approx 34 \%)$ while Susceptible, Exposed, and Recovered population compartments seem to be plausible. We used our framework to create three intervention scenarios and test the effects of different mitigation options for the epidemic.

\section{Scenario 1 - Vaccination}

In this intervention scenario, a certain percentage of the population is vaccinated before the start of the first infection time (day 26). The vaccinated population is randomly sampled from the entire population, is assumed to be in the recovered stage once vaccinated (not susceptible to disease). We tested three different values $(10 \%, 30 \%$, and 50\%) for the percentages of vaccinated people with ten replications. Fig. 6 shows the comparative results among these interventions against the baseline. It is clear that vaccination even at a low level reduces the peak of the epidemic. That is, $10 \%$ vaccination reduces the peak of the epidemic by $\approx 13 \% ; 30 \%$ vaccination reduces the peak of the epidemic by $\approx 37 \%$, and $50 \%$ vaccination reduces the peak of the epidemic by $\approx 62 \%$. Overall, vaccination appears to be a plausible strategy to cope with such epidemics. 


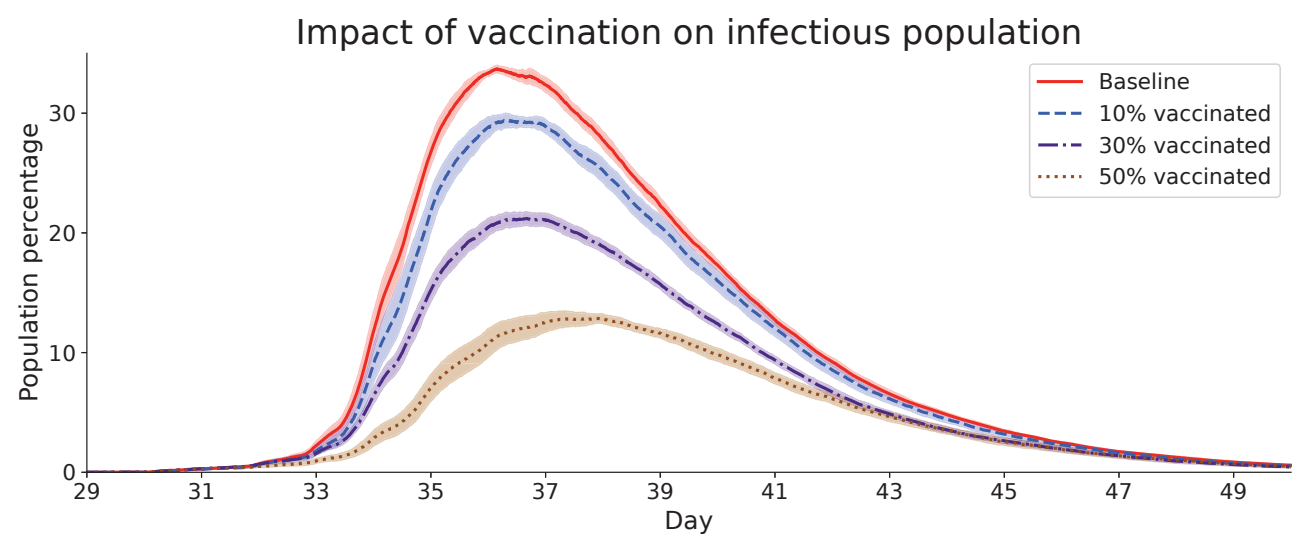

Figure 6: The impact of different percentages of vaccinated people on the infectiousness of the disease. The shaded areas show 95\% confidence band coverage while the lines within the shaded areas indicate the average values.

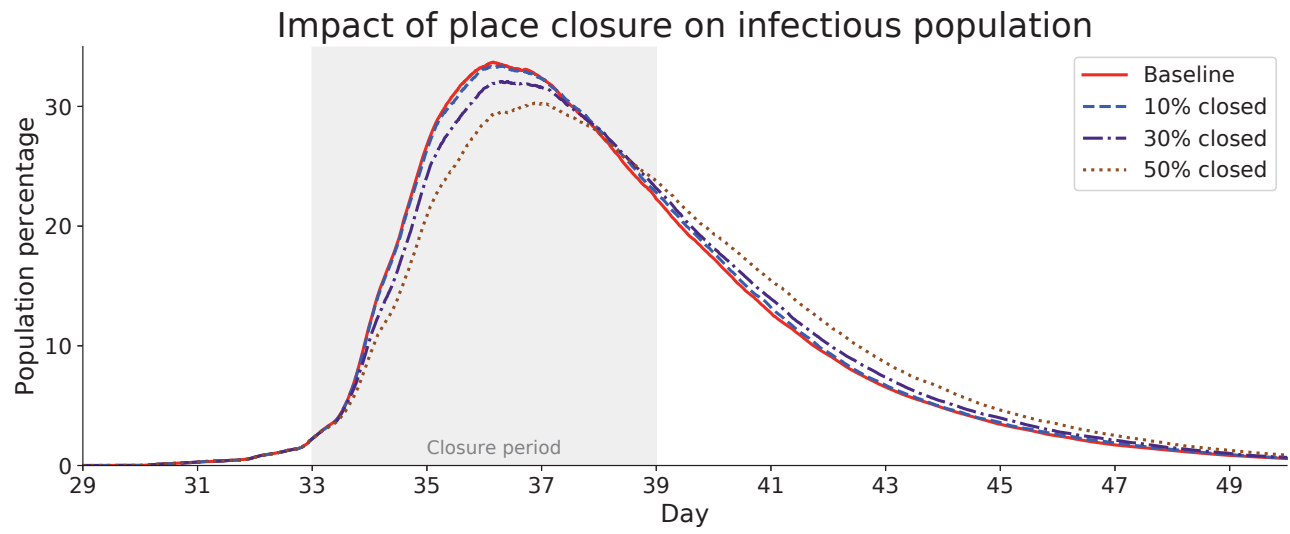

Figure 7: The impact of closing different percentage of public places on the infectiousness of the disease. Lines indicate the average values. Confidence bands are hidden because they overlap on each other making it challenging to read.

\section{Scenario 2 - Place Closure}

In this intervention scenario, the city ordered a certain percentage of meeting venues to be closed for a certain number of days. As a result, people who aim to visit those places spend their time in their current location for the length of the planned visit. Similar to the vaccination case, the choice of places to be closed are randomly sampled. With ten replications, we tested $10 \%, 30 \%$, and $50 \%$ of places closed for six days as indicated in Fig. 7. Unlike the vaccination case, place closures do not help to mitigate the peak of the infectious population as much. Place closures only reduced the epidemic peak by $\approx 1 \%, \approx 5 \%$, and $\approx 10 \%$, respectively. Further, there were more infectious cases, compared to the baseline, right after the closure period ended. As a result, considering the cost to businesses against very limited gain, place closures doesn't appear to be a feasible solution. We tried changing the closure period to three days, but our results were almost identical to the 6-day closure intervention.

\section{Scenario 3 - Stay Home Order}

The third and final intervention scenario is the order from the city that asks people to stay home when they know that they are sick. In this scenario, we randomly sampled $10 \%, 30 \%$, and $50 \%$ of the population to 


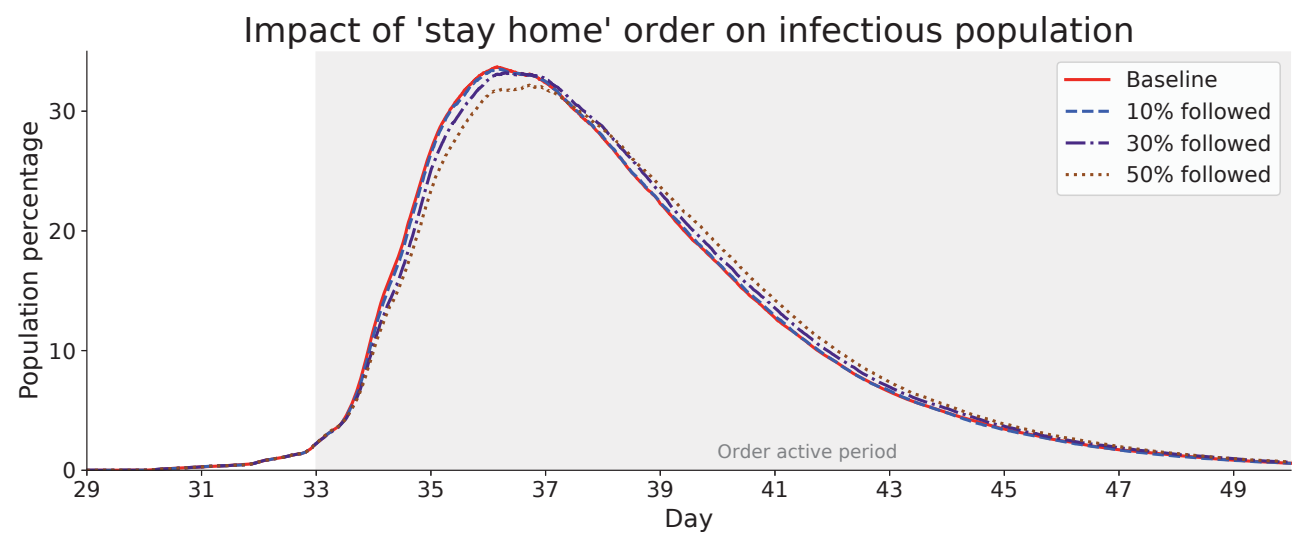

Figure 8: The impact of stay home orders on the infectious population. Lines indicate the average values. Confidence bands are hidden because they overlap on each other making it challenging to read.

follow such orders starting with day 33 as shown in Fig. 8. Again, we conducted ten replications to capture uncertainties caused by stochastic processes in the model. Results indicate that these orders are even more ineffective than place closures. When $10 \%, 30 \%$, and $50 \%$ of people follow such orders, the peak of the epidemic only reduced by $\approx 0.6 \%, \approx 1.5 \%$, and $\approx 7.5 \%$, respectively. These results show that even worse than place closure, asking people to stay home is not an effective strategy.

To summarize, vaccination appears to be the most effective method, reducing the peak of the epidemic compared to the other two. Stay home and place closure measures don't seem to work based on our interpretation. We want to note that our model does not preclude people to work or does not close workplaces.

\section{CONCLUSION}

In spite of the importance of experimentation, it has been conducted ad-hoc and involves manual code adjustments which are time-consuming and error-prone. In this paper, we introduced a framework that facilitates changing agent-based model states while running the simulation to explore alternative worlds in a semi-automated manner. Such a framework allows the simulation user to explore different policy options rapidly with minimal effort. To demonstrate the effectiveness of our framework, we implemented an agentbased urban model that simulates the spread of influenza-like diseases based on the framework. No code for experimentation is included in the model. We successfully dispensed prescriptions for a given problem through experimentation.

To reach a persuasive conclusion from the outcome of experimentation, numerous runs and substantial resources such as computing power are required. Our future work is to extend our framework to leveraging cloud computing. We further aim to extend our framework to facilities the automated comparison between different "what-if" instances to support users in exploring relationship and causality between prescribed changes and observable simulation results.

\section{ACKNOWLEDGMENTS}

This project is sponsored by the Defense Advanced Research Projects Agency (DARPA) under cooperative agreement No.HR00111820005. The content of the information does not necessarily reflect the position or the policy of the Government, and no official endorsement should be inferred. 


\section{REFERENCES}

Apolloni, A., V. S. A. Kumar, M. V. Marathe, and S. Swarup. 2009, Dec. "Computational Epidemiology in a Connected World". Computer vol. 42 (12), pp. 83-86.

Balcan, D., H. Hu, B. Goncalves, P. Bajardi, C. Poletto, J. J. Ramasco, D. Paolotti, N. Perra, M. Tizzoni, W. Van den Broeck, V. Colizza, and A. Vespignani. 2009, Sep. "Seasonal transmission potential and activity peaks of the new influenza $\mathrm{A}(\mathrm{H} 1 \mathrm{N1})$ : a Monte Carlo likelihood analysis based on human mobility". BMC Medicine vol. 7 (1), pp. 45.

Barrett, C. L., K. R. Bisset, S. G. Eubank, X. Feng, and M. V. Marathe. 2008, Nov. "EpiSimdemics: An efficient algorithm for simulating the spread of infectious disease over large realistic social networks". In SC '08: Proceedings of the 2008 ACM/IEEE Conference on Supercomputing, pp. 1-12.

Carley, K. M., D. B. Fridsma, E. Casman, A. Yahja, N. Altman, L.-C. Chen, B. Kaminsky, and D. Nave. 2006. "BioWar: scalable agent-based model of bioattacks". IEEE Transactions on Systems, Man, and Cybernetics-Part A: Systems and Humans vol. 36 (2), pp. 252-265.

Crooks, A. T., and A. B. Hailegiorgis. 2014. "An agent-based modeling approach applied to the spread of cholera". Environmental Modelling \& Software vol. 62, pp. 164-177.

Farmer, J. D., and D. Foley. 2009. “The economy needs agent-based modelling”. Nature vol. 460 (7256), pp. 685.

Grow, A. 2017. Regression Metamodels for Sensitivity Analysis in Agent-Based Computational Demography, pp. 185-210. Springer International Publishing.

Hybinette, M., and R. Fujimoto. 1997. "Cloning: a novel method for interactive parallel simulation”. In wsc, pp. 444-451. IEEE.

Hybinette, M., and R. M. Fujimoto. 2001. "Cloning parallel simulations”. ACM Transactions on Modeling and Computer Simulation (TOMACS) vol. 11 (4), pp. 378-407.

Kim, J.-S., H. Kavak, and A. Crooks. 2018. "Procedural city generation beyond game development". SIGSPATIAL Special vol. 10 (2), pp. 34-41.

Kleijnen, J. P. 2005. "An overview of the design and analysis of simulation experiments for sensitivity analysis". European Journal of Operational Research vol. 164 (2), pp. 287-300.

Kumar, S., J. J. Grefenstette, D. Galloway, S. M. Albert, and D. S. Burke. 2013. "Policies to reduce influenza in the workplace: impact assessments using an agent-based model". American journal of public health vol. 103 (8), pp. 1406-1411.

Lamperti, F., A. Roventini, and A. Sani. 2018. "Agent-based model calibration using machine learning surrogates". Journal of Economic Dynamics and Control vol. 90, pp. 366-389.

Li, X., W. Cai, and S. J. Turner. 2017, May. "Cloning Agent-Based Simulation”. ACM Trans. Model. Comput. Simul. vol. 27 (2), pp. 15:1-15:24.

Ligmann-Zielinska, A., D. B. Kramer, K. Spence Cheruvelil, and P. A. Soranno. 2014, 10. "Using Uncertainty and Sensitivity Analyses in Socioecological Agent-Based Models to Improve Their Analytical Performance and Policy Relevance”. PLOS ONE vol. 9 (10), pp. 1-13.

Luke, S., C. Cioffi-Revilla, L. Panait, K. Sullivan, and G. Balan. 2005. "Mason: A multiagent simulation environment". Simulation vol. 81 (7), pp. 517-527.

Malleson, N., A. Heppenstall, and L. See. 2010. "Crime reduction through simulation: An agent-based model of burglary”. Computers, environment and urban systems vol. 34 (3), pp. 236-250. 
Nsoesie, E. O., J. S. Brownstein, N. Ramakrishnan, and M. V. Marathe. 2013. "A systematic review of studies on forecasting the dynamics of influenza outbreaks". Influenza and Other Respiratory Viruses vol. 8 (3), pp. 309-316.

Perez, L., and S. Dragicevic. 2009. "An agent-based approach for modeling dynamics of contagious disease spread". International journal of health geographics vol. 8 (1), pp. 50.

Pires, B., and A. T. Crooks. 2017. "Modeling the emergence of riots: A geosimulation approach". Computers, Environment and Urban Systems vol. 61, pp. 66-80.

Prasanna, D. R. 2009. Dependency Injection. 1st ed. Greenwich, CT, USA, Manning Publications Co.

Riley, S. 2007. "Large-Scale Spatial-Transmission Models of Infectious Disease". Science vol. 316 (5829), pp. 1298-1301.

Roche, B., J. M. Drake, and P. Rohani. 2011, Mar. "An Agent-Based Model to study the epidemiological and evolutionary dynamics of Influenza viruses". BMC Bioinformatics vol. 12 (1), pp. 87.

Thiele, J. C., W. Kurth, and V. Grimm. 2014. "Facilitating Parameter Estimation and Sensitivity Analysis of Agent-Based Models: A Cookbook Using NetLogo and 'R"'. Journal of Artificial Societies and Social Simulation vol. 17 (3), pp. 11.

Yoginath, S. B., and K. S. Perumalla. 2018, January. "Scalable Cloning on Large-Scale GPU Platforms with Application to Time-Stepped Simulations on Grids”. ACM Trans. Model. Comput. Simul. vol. 28 (1), pp. 5:11-5:26.

\section{AUTHOR BIOGRAPHIES}

JOON-SEOK KIM is a Postdoctoral Research Fellow in Department of Geography and Geoinformation Science at George Mason University. His research interests lie in spatial and spatiotemporal databases, geospatial simulation, and privacy protection. His email address is jkim258@gmu.edu.

HAMDI KAVAK is a Research Associate in the Department of Geography and Geoinformation Science at George Mason University. His research focuses on data-driven methods to study human behavior in the areas of cybersecurity and urban systems. His email address is hkavak@gmu.edu.

UMAR MANZOOR is a Postdoctoral Research Fellow in the Department of Computer Science at Tulane University. His research focuses on multi-agent modelling and simulation. His email address is umanzoor@tulane.edu.

ANDREAS ZÜFLE is an assistant professor at the Department of Geography and Geoinformation Science at George Mason University (GMU). Dr. Züfle's research expertise includes big spatial data, spatial data mining, social network mining, and uncertain database management. His email address is azufle@gmu.edu. 